\title{
Correlação entre as escalas visual analógica, de Melbourne e filamentos de Von Frey na avaliação da dor pós-operatória em cadelas submetidas à ovariossalpingohisterectomia
}

\author{
Correlation between visual analog scale, Melbourne pain scale and Von Frey filaments in \\ post-operatory pain evaluation in bitches submitted to ovaryhysterectomy
}

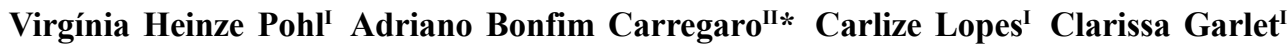 \\ Jenifer Santana Marques ${ }^{\mathrm{I}}$
}

RESUMO

A avaliação da dor em animais necessita da utilização de escalas de avaliação, que dependem da interpretação realizada por observadores. O objetivo do presente estudo foi avaliar a correlação entre a escala visual analógica (EVA), escala de Melbourne e os filamentos de Von Frey, na avaliação da dor pós-operatória em 42 cadelas adultas e saudáveis, submetidas à ovariossalpingohisterectomia (OSH). A dor pós-operatória foi avaliada por dois observadores cegos aos tratamentos analgésicos, em intervalos de uma hora, utilizando a EVA, a escala de Melbourne e os filamentos de Von Frey, aplicados ao redor da incisão cirúrgica. Foram considerados como critérios para realização da analgesia resgate uma pontuação de $50 \mathrm{~mm}$ na EVA ou de 13 pontos na escala de Melbourne. A EVA revelou-se a escala mais sensivel, uma vez que $100 \%$ dos animais receberam resgate seguindo esse método. Os valores obtidos na EVA e na escala de Melbourne determinaram boa correlação, com $r=0,74$, o que não ocorreu com os filamentos de Von Frey $(r=-0,18)$. Já a correlação entre a escala de Melbourne e os filamentos de Von Frey foi de -0.37. Apesar de a EVA e a escala de Melbourne apresentarem boa correlação, sugere-se que se considere uma pontuação menor na escala de Melbourne como critério para administração de analgesia resgate.

Palavras-chave: dor, analgesia, métodos de avaliação, cães.

\section{ABSTRACT}

Pain evaluation in animals requires the use of evaluation scales, which depend on the interpretation made by observers. The present study aimed to evaluate the correlation between visual analog scale (VAS), Melbourne pain scale and
Von Frey filaments in the evaluation of post-operatory pain in fourty two healthy adult bitches submited to ovariohysterectomy. Post-operatory pain was evaluated by two observers unaware of the analgesic protocol used, in one-hour intervals, using VAS, Melbourne pain scale and Von Frey filaments, applied around the surgical wound. A score of $50 \mathrm{~mm}$ in VAS or 13 points in the Melbourne pain scale were considered as the criterion for analgesic administration. VAS and Melbourne pain scale showed a good correlation, with $r=0.74$. Correlation between VAS and Von Frey filaments was weak $(r=-0.18)$. Correlation between Melbourne pain scale and Von Frey filaments was -0.37. VAS revealed to be the most sensitive scale. VAS and Melbourne pain scale showed a good correlation degree. It was concluded that a lower score in the Melbourne pain scale can be considered as criterion for analgesic administration.

Key words: pain, analgesia, evaluation methods, dogs.

\section{INTRODUÇÃO}

Pode ser extremamente difícil avaliar o grau da dor vivenciada por animais e a habilidade destes em lidar com a dor (MATHEWS, 2000). Essa avaliação depende da análise e interpretação comportamentais realizadas por um ou mais observadores, resultando na necessidade de desenvolvimento de métodos ou escalas de avaliação que produzam resultados mais objetivos. Apesar disso, a avaliação da dor aguda cirúrgica e traumática em cães e gatos tem recebido crescente atenção (GAYNOR \& MUIR, 2009).

'Departamento de Clínica de Pequenos Animais, Centro de Ciências Rurais (CCR), Universidade Federal de Santa Maria (UFSM), Camobi, Santa Maria, RS, Brasil.

"Faculdade de Zootecnia e Engenharia de Alimentos, Universidade de São Paulo (USP), Av. Duque de Caxias Norte, 225, 13635900, Pirassununga, SP, Brasil. E-mail: carregaro@usp.br. *Autor para correspondência. 
A escala visual analógica (EVA) é um sistema de pontuação semiobjetivo utilizado para quantificar a intensidade da dor (GAYNOR \& MUIR, 2009). AEVAé tipicamente uma linha reta horizontal de $100 \mathrm{~mm}$ de comprimento, que descreve a intensidade dolorosa (ex.: nenhuma dor; pior dor possível) em cada uma das extremidades da linha. Essa escala tem sido extensivamente utilizada na medicina, sendo geralmente preenchida pelo próprio paciente. Seu uso na medicina veterinária depende de um observador para identificar e interpretar comportamentos de dor no paciente, tendo sua precisão afetada pela variabilidade entre observadores (HOLTON et al., 1998).

A escala de dor da Universidade de Melbourne (FIRTH \& HALDANE, 1999) é uma escala baseada em respostas comportamentais e fisiológicas específicas, incluindo descritores múltiplos em seis categorias. Tais categorias contam com observações comportamentais que limitam assim a interpretação e propensão do observador, além de avaliar mudanças no comportamento ou na conduta, aumentando a sensibilidade da escala. No entanto, até o presente momento, sua validação ainda é limitada (GAYNOR \& MUIR, 2009).

Os filamentos de Von Frey são utilizados para determinar parâmetros de origem tátil, mecânica e para quantificar objetivamente processos nociceptivos, já que a deformação de suas hastes pode ser transformada em parâmetros de força com unidade em gramas, fazendo com que a avaliação do processo doloroso deixe de ser subjetivo e passe a ter um caráter mais direto (VALADÃO et al., 2002). Seu uso foi considerado um método confiável de quantificação da hiperalgesia pós-operatória em homens (ILKJAER et al., 1998) e ratos (BRENNAN et al., 1996).

A EVA e escalas numéricas, como a escala de Melbourne, foram utilizadas com sucesso na avaliação da dor em cães (CONZEMIUS et al., 1997; FIRTH \& HALDANE, 1999; GRISNEAUX et al., 1999). Embora essas escalas pareçam ter precisão e acurácia similares, mesmo quando utilizadas por múltiplos avaliadores, em humanos, a EVA foi relatada como sendo a escala mais sensível (MANNE et al., 1992). No entanto, SELMI et al. (2009) encontraram alta correlação entre a EVA e a escala de Melbourne em cadelas submetidas à OSH. Os filamentos de Von Frey foram utilizados com sucesso na avaliação de hiperalgesia pós-operatória em cadelas submetidas à $\mathrm{OSH}$ (LASCELLES et al., 1997), embora tenham demonstrado baixa correlação com a EVA.

O objetivo do presente estudo foi avaliar a correlação entre a EVA, escala de Melbourne e os filamentos de Von Frey, na avaliação da dor pósoperatória em cadelas submetidas à OSH.

\section{MATERIAL E MÉTODOS}

Foram utilizadas 42 cadelas adultas, sem raça definida, com peso médio de $14,9 \pm 4,0 \mathrm{~kg}^{-1}$, as quais foram submetidas à $\mathrm{OSH}$. O projeto foi devidamente aprovado pelo Comitê de Ética e Bem-Estar Animal da Instituição de origem, sob o número 01/2009.

Os animais utilizados foram provenientes de uma sociedade protetora de animais, sendo encaminhados para adoção após a realização do procedimento. Eles foram incluídos no estudo após serem considerados hígidos, por meio de exames clínico e laboratorial (hemograma, contagem de plaquetas e bioquímica hepática e renal). Também passaram por um período de adaptação de uma semana ao ambiente experimental e ao contato com os observadores antes da realização do procedimento cirúrgico. O protocolo anestésico utilizado para as castrações foi composto de medicação pré-anestésica com $0,1 \mathrm{mg} \mathrm{kg}^{-1}$ de acepromazina e manutenção com a associação de lidocaína $1 \%$ com vasoconstritor $(\mathrm{c} / \mathrm{v})$ e diferentes tipos de agonistas alfa $\mathrm{a}_{2}$ adrenérgicos $\left(0,25 \mathrm{mg} \mathrm{kg}^{-1}\right.$ de xilazina, $5 \mu \mathrm{g} \mathrm{kg}^{-1}$ de clonidina, $10 \mu \mathrm{g} \mathrm{kg}^{-1}$ de romifidina, $30 \mu \mathrm{g} \mathrm{kg}^{-1} \mathrm{de}$ detomidina ou $2 \mu \mathrm{g} \mathrm{kg}^{-1}$ de dexmedetomidina), perfazendo um volume final de $1 \mathrm{~mL} 4 \mathrm{~kg}^{-1}$, sendo complementada com isofluorano quando necessário (estudo paralelo). Todos os procedimentos cirúrgicos foram realizados pela mesma equipe.

A avaliação da analgesia pós-operatória foi realizada por dois observadores treinados, cegos aos protocolos de analgesia utilizados durante a anestesia. A avaliação foi feita através da EVA, seguida pela escala de Melbourne e avaliação da sensibilidade na linha de incisão, utilizando-se os filamentos de Von Frey, em intervalos de uma hora, iniciando-se duas horas após a administração da anestesia epidural.

$\mathrm{Na}$ véspera do procedimento, os animais foram submetidos ao exame clínico, mensurando-se a frequência cardíaca (FC), em batimentos por minuto, e frequência respiratória $\left(\mathrm{F}_{\mathrm{R}}\right)$, em movimentos por minuto, pressão arterial sistólica (PAS) ${ }^{\mathrm{a}}$, em $\mathrm{mmHg}$, e temperatura corporal $\left(\mathrm{T}^{\circ} \mathrm{C}\right)^{\mathrm{b}}$, em graus Celsius, os quais foram considerados como parâmetros basais para a avaliação pós-operatória através da escala de Melbourne.

Para avaliação através da EVA, utilizou-se uma linha reta com $100 \mathrm{~mm}$ de comprimento, sendo uma das extremidades considerada como ausência de dor e a outra como a pior dor possível. Observou-se o comportamento do animal na gaiola e solto na sala (atividade, status mental, postura, vocalização), sendo em seguida assinalado, na linha, pelos observadores, o valor correspondente ao grau de dor. Posteriormente, foi realizada a média de pontos entre eles.

Ciência Rural, v.41, n.1, jan, 2011. 
Para a avaliação através da escala de Melbourne, além da observação do comportamento do animal (atividade, status mental, postura, vocalização) e palpação da incisão cirúrgica, mensuraram-se os valores de FC, $\mathrm{F}_{\mathrm{R}}$, PAS e temperatura retal, bem como a presença de salivação e dilatação pupilar, pontuadas conforme as categorias avaliadas na escala previamente descrita por FIRTH \& HALDANE (1999), em que enquadra valores entre zero (ausência de dor) e 27 (pior dor possível).

Os filamentos de Von Frey ${ }^{\mathrm{c}}$ foram aplicados em quatro pontos (cranial, caudal, lateral esquerdo e direito) ao redor da incisão, a uma distância de $0,5 \mathrm{~cm}$ desta. Iniciava-se com o filamento mais fino e, caso não houvesse resposta, era utilizado o próximo filamento e, assim, sucessivamente, até a obtenção de uma resposta aversiva (contração do abdome, vocalização, movimentação da cabeça em direção ao abdome). Desse modo, registrou-se o valor do filamento que foi capaz de induzir a resposta.

Caso fossem imputados valores acima de $50 \mathrm{~mm}$ na EVA ou acima de 13 pontos na escala de Melbourne, era realizada analgesia resgate com $0,5 \mathrm{mg}$ $\mathrm{kg}^{-1}$ de morfina ${ }^{\mathrm{d}} \mathrm{IM}$ e $0,2 \mathrm{mg} \mathrm{kg}^{-1}$ de meloxicam ${ }^{\mathrm{e}}$ IM. Uma hora após a administração dos analgésicos, os animais eram submetidos a uma nova avaliação, sendo que todos os momentos foram utilizados nas comparações entre os métodos descritos.

Para a análise estatística ${ }^{\mathrm{f}}$ dos dados, utilizouse correlação de Pearson, avaliando-se as metodologias empregadas em pares. Para isso, as correlações ( $r$ ) foram determinadas, podendo variar entre $-1,0$, ou seja, a máxima correlação inversamente proporcional e 1,0, a qual atesta máxima correlação diretamente proporcional. Destaca-se que uma boa correlação deve ser $<-0,7 \mathrm{ou}>0,7$.

\section{RESULTADOS}

Os procedimentos cirúrgicos foram realizados em $27 \pm 11,8 \mathrm{~min}$. O total de avaliações de cada um dos métodos obtidos nos 42 animais foi de 135. Em virtude da análise estatística realizada, para as comparações envolvendo os filamentos de Von Frey, foram removidas 62 avaliações, nas quais os animais não reagiram a nenhum dos filamentos.

AEVA revelou-se a escala mais sensível, sendo que $100 \%$ dos animais receberam analgesia resgate baseando-se nessa metodologia. Observouse também aumento da pontuação na escala de Melbourne, porém sem atingir a pontuação necessária, conforme literatura para administração de analgésicos.
Os valores obtidos na EVA e na escala de Melbourne determinaram boa correlação, com $\mathrm{r}=0,74$ (Figura 1), o que não foi observado com os filamentos de Von Frey e a EVA $(r=-0,18)$ (Figura 2) e a escala de Melbourne ( $\mathrm{r}=-0.37$ ) (Figura 3), que foram consideradas fracas.

\section{DISCUSSÃO}

$\mathrm{O}$ presente estudo utilizou a OSH como modelo para a comparação das metodologias empregadas para a avaliação da dor pós-operatória, pois ela já provou ser útil no estudo do mecanismo e tratamento desse tipo de dor (GONZALES et al., 2000).

Dentre os métodos utilizados para avaliar a dor pós-operatória, a EVA revelou-se o método mais sensível. Na medicina, observa-se resultado semelhante, sendo que a EVA revela-se mais sensível quando comparada às escalas numéricas utilizadas por múltiplos avaliadores (MANNE et al., 1992), embora na medicina veterinária a variabilidade entre observadores seja capaz de alterar sua precisão (HOLTON et al., 1998). No entanto, o aspecto interpretativo da EVA pode ser um ponto positivo, permitindo aos avaliadores maior precisão devido ao maior grau de variação, não estando limitada à variação dentro de categorias restritas (QUINN et al., 2007). Em gatos, a EVA e a resposta à palpação demonstraram ser mais sensíveis do que a utilização de escalas descritivas na determinação da presença de dor pós-operatória (CAMBRIDGE et al., 2000).

A escala de Melbourne, embora tenha demonstrado ser eficiente no presente estudo, não foi tão sensível quanto a EVA. As escalas numéricas, entre as quais se pode incluir a escala de Melbourne, utilizam

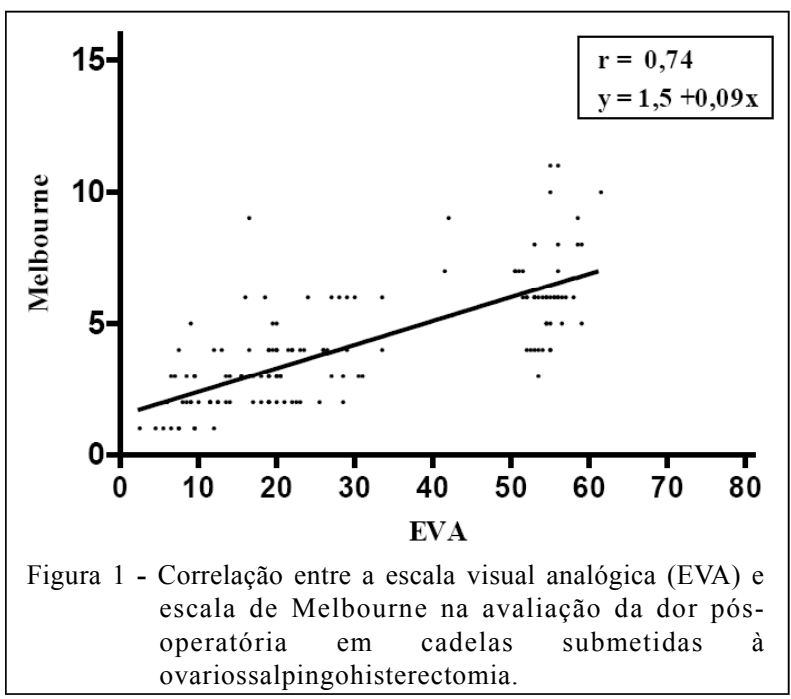




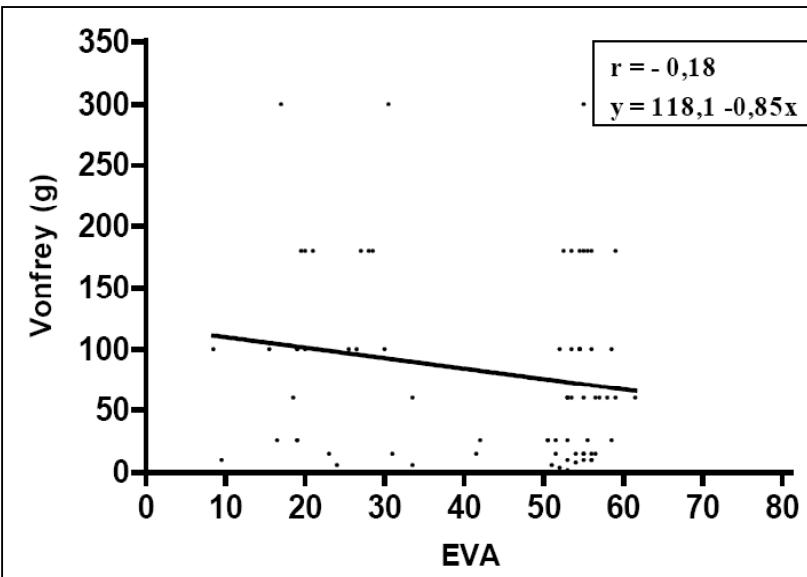

Figura 2 - Correlação entre a escala visual analógica (EVA) e os filamentos de Von Frey na avaliação da dor pósoperatória em cadelas submetidas à ovariossalpingohisterectomia.

avaliações comportamentais e também parâmetros fisiológicos. No entanto, a utilização de parâmetros fisiológicos, como frequência cardíaca, frequência respiratória e dilatação de pupilas, os quais são avaliados pela escala de Melbourne, não demonstraram serem indicadores úteis de dor em cães hospitalizados (HOLTON et al., 1998b).

Embora os filamentos de Von Frey tenham sido utilizados com sucesso para avaliação de dor pósincisional em cães (VALADÃO et al., 2002; DUQUE et al., 2004) e equinos (RÉDUA et al., 2002), destaca-se que esses modelos utilizaram dor superficial. No caso da dor proveniente da $\mathrm{OSH}$, além da incisão superficial, tem-se uma intensidade importante proveniente da parede muscular e da cavidade abdominal, sendo que a avaliação com os filamentos de Von Frey não

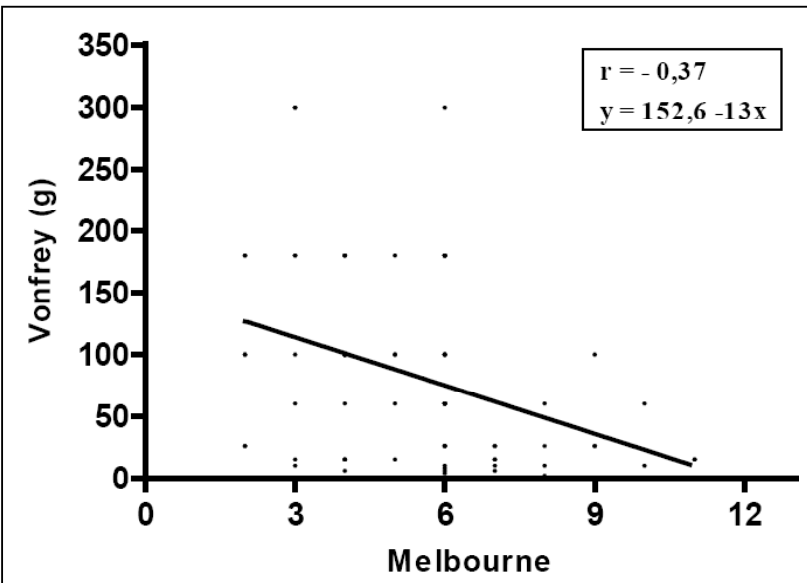

Figura 3 - Correlação entre a escala de Melbourne e os filamentos de Von Frey na avaliação da dor pós-operatória em cadelas submetidas à ovariossalpingohisterectomia. conseguiu refletir completamente a dor dos animais, tendo fraca sensibilidade quando comparada à mensuração qualitativa da dor (EVA) em cadelas submetidas à $\mathrm{OSH}$.

Observou-se no presente estudo que houve boa correlação entre a escala de Melbourne e a EVA na avaliação de dor pósoperatória em cadelas submetidas à $\mathrm{OSH}$, resultado semelhante ao obtido por SELMI et al. (2009), os quais determinaram alta correlação entre a escala de Melbourne e a EVA em cadelas submetidas à $\mathrm{OSH}$. Em cães submetidos a cirurgias ortopédicas, também houve alta correlação entre a EVA e uma escala numérica (CONZEMIUS et al., 1997).

No entanto, apesar da boa correlação entre as duas escalas, nenhum dos animais obteve pontuação necessária para administração de analgesia resgate através da escala de Melbourne, sendo que a pontuação máxima alcançada nessa escala foi de 11 pontos. Em cadelas submetidas à OSH por meio de videolaparoscopia e avaliadas através da escala de Melbourne, a pontuação manteve-se abaixo de seis pontos (BAKHTIARI et al., 2006). Em cadelas submetidas à OSH, a pontuação da escala de Melbourne manteve-se abaixo de nove pontos (SELMI et al., 2009). Esses resultados sugerem que, quando a escala de Melbourne for o método empregado para avaliação da dor pós-operatória em animais submetidos à $\mathrm{OSH}$, deve-se considerar uma pontuação menor como indicador para administração da analgesia de resgate. O presente estudo considerou a pontuação a partir de 13 como valor para administração de analgesia resgate, sendo o valor intermediário entre 0 e 27 (maior pontuação possível), relacionando-se com o valor considerado na EVA, que foi de $50 \mathrm{~mm}$ (valor intermediário entre 0 e $100 \mathrm{~mm}$ ). No entanto, o valor de 50mm na EVA correlacionou-se com uma pontuação entre 6 e 7 na escala de Melbourne, podendo ser considerado assim, como a pontuação necessária para administração da analgesia resgate em cadelas submetidas à $\mathrm{OSH}$.

Os filamentos de Von Frey, por sua vez, não determinaram boa correlação com a EVA ou com a escala de Melbourne. Até o presente momento, não foram encontrados artigos avaliando a correlação entre os filamentos de Von Frey e a escala de Melbourne. No entanto, em cadelas submetidas à $\mathrm{OSH}$, os filamentos de Von Frey demonstraram ter fraca correlação com a EVA (LASCELLES et al., 1997), resultado semelhante ao obtido no presente estudo.

Ciência Rural, v.41, n.1, jan, 2011. 


\section{CONCLUSÃO}

A EVA demonstrou ser a melhor metodologia empregada para avaliação da dor pósoperatória em cadelas submetidas à OSH. Apesar de a escala de Melbourne ter boa correlação com a EVA, a pontuação necessária para a administração de analgesia resgate deve ser reconsiderada. Os filamentos de Von Frey não se correlacionaram significativamente com os outros métodos utilizados, apresentando-se pouco efetivos para demonstrar adequadamente a dor pósoperatória em cadelas submetidas à OSH.

\section{FONTES DE AQUISIÇÃO}

a - Doppler vascular DV - 10, Microem ${ }^{\circledast}$ Prod. Méd. Ltda, Ribeirão Preto, SP, Brasil.

b - BD ${ }^{\circledR}$, São Paulo, SP, Brasil.

c - Von Frey Anesthesiometer, IITC Inc. Life Science, California, USA.

d - Dimorf®, Cristália Prod. Quím.Farm. LTDA, Itapira, SP, Brasil.

e - Maxicam ${ }^{\circledR} 2 \%$, Ouro Fino Saúde Animal, Cravinhos, SP, Brasil.

f - GraphPad Prism, GraphPad Software Inc, San Diego, California, USA.

\section{REFERÊNCIAS}

BAKHTIARI, J. et al. Clinical evaluation of elective laparoscopic ovariohysterectomy in dog. Iranian Journal of Veterinary Surgery, v.1, n.1, p.15-20, 2006. Disponível em: $<$ http:// www.sid.ir/En/VEWSSID/J_pdf/115920060102.pdf>. Acesso em: 23 jun. 2009.

BRENNAN, T.J. et al. Characterization of a rat model of incisional pain. Pain, v.64, n.3, p.493-501, 1996. Disponível em: <http:// www.sciencedirect.com/science? ob=MImg\& imagekey $=$ B6T0K3W0NBP2-16-1\&_cdi $=4865 \&$ user $=687358 \&$ \& ii $=030439599$ $5014411 \&$ origin $=$ browse \& coverDate $=03 \% 2$ F31\%2F1996\&_sk=999359996\&view $=\mathrm{c} \& w \mathrm{w}$ chp $=$ dGLzVtbzSkWA\&md5 $=64$ ef57041824cd7b05c $8 \mathrm{f} 1 \mathrm{~b} 71 \mathrm{a} 992 \mathrm{~d} 7 \mathrm{f} \& \mathrm{ie}=/$ sdarticle.pdf $>$. Acesso em: 20 jul. 2009. doi: 10.1016/03043959(95)01441-1.

CAMBRIDGE, A.J. et al. Subjective and objective measurements of postoperative pain in cats. Journal of the American Veterinary Medical Association, v.217, n.5, p.685-690, 2000. Disponível em: <http://avmajournals.avma.org/doi/pdf/ 10.2460/javma.2000.217.685>. Acesso em: 25 jun. 2009. doi: $10.2460 /$ javma.2000.217.685.

CONZEMIUS, M.G. et al. Correlation between subjective and objective measures to determine severity of postoperative pain in dogs. Journal of the American Veterinary Medical Association, v.210, p.1619-1622, 1997.

DUQUE, J.C. et al. Pre-emptive epidural ketamine or S(+)ketamine in post-incisional pain in dogs: a comparative study. Veterinary Surgery, v.33, n.4, p.361-367, 2004. Disponível em: $<$ http://onlinelibrary.wiley.com/doi/10.1111/j.1532950X.2004.04052.x/pdf>. Acesso em: 3 jul. 2009. doi: 10.1111/j.1532-950X.2004.04052.x.
FIRTH, A.M.; HALDANE, S.L. Development of a scale to evaluate postoperative pain in dogs. Journal of the American Veterinary Medical Association, v.214, p.651-659, 1999.

GAYNOR, J.S.; MUIR, W.W. Manual de controle da dor em Medicina Veterinária. 2.ed. São Paulo: MedVet, 2009. $643 p$.

GONZALES, M.I. et al. Ovaryhysterectomy in the rat: a model of surgical pain for evaluation of pre-emptive analgesia? Pain, v.88, n.1, p.79-88, 2000. Disponível em: $<$ http://www.sciencedirect.com/ science?_ob=MImg\&_imagekey=B6T0K-41BV7KR-BM \&_cdi $=4865 \&$ \& u e r $=687358 \&$ \& i i $=$ S 03043 $95900003092 \&$ origin $=$ browse \& zone $=$ rslt list $\_$item $\&$ coverDate $=1$ 0\%2F01\%2F2000\&_sk $=999119998 \& \mathrm{w} c h \mathrm{p}=\overline{\mathrm{d}} \mathrm{FLbVzW}-$ zSkzk\&md5=821 edc0cdd6c394befe561d040957104\&ie $=/$ sdarticle.pdf $>$. Acesso em: 8 jul. 2009. doi: S0304-3959(00)00309-2.

GRISNEAUX, E. et al. Comparison of ketoprofen and carprofen prior to orthopedic surgery for control of postoperative pain in dogs. Journal of the American Veterinary Medical Association, v.215, p.1105-1110, 1999.

HOLTON, L.L. et al. Comparison of three methods used for assesment of pain in dogs. Journal of the American Veterinary Medical Association, v.212, p.61-66, 1998.

HOLTON, L.L. et al. Relationship between physiological factors and clinical pain in dogs scored using a numerical rating scale. Journal of Small Animal Practice, v.39, n.10, p.469474, 1998b. Disponível em: <http://onlinelibrary.wiley.com/ doi/10.1111/j.1748-5827.1998.tb03681.x/pdf>. Acesso em: 11 jun. 2009. doi: 10.1111/j.1748-5827.1998.tb03681.x

ILKJAER, S. et al. Effect of intravenous ketamine in combination with epidural bupivacaine or epidural morphine on postoperative pain and tenderness after renal surgery. British Journal of Anesthesia, v.81, p.707-712, 1998. Disponível em: <http://bja.oxfordjournals.org/content/81/5/ 707.full.pdf+html $>$. Acesso em: 30 jun. 2009. Doi: 10.1093/ bja/81.5.707.

LASCELLES, B.D.X. et al. Post-operative central hypersensitivity and pain: the pre-emptive value of pethidine for ovariohysterectomy. Pain, v.73, p.461-471, 1997. Disponível em: <http:// www.sciencedirect.com/science?_ob=MImg\&_imagekey=B6T0K3 RSFPRG-S-5\&_cdi $=4865 \&$ \&user $=687358 \&$ \&pii $=\mathrm{S} 03043$ $95997001413 \&_{-}$origin $=$browse\&_zone $=$rslt_lis t_i t e m \&_c o ver D a t e = 12\%2 F $31 \% 2 \mathrm{~F} 1$ $997 \&$ \& k $=999269996 \& \mathrm{w} \mathrm{ch} \mathrm{p}=\mathrm{d} \mathrm{G} \mathrm{L} \mathrm{z} \mathrm{V} \mathrm{z} \mathrm{z} \mathrm{-}$ zSkWA \& md5=03f9ce99713b22fe921f94f24d8649c6\&ie=/ sdarticle.pdf $>$. Acesso em: 5 jul. 2009. doi: S0304-3959(97)00141-3.

QUINN, M.M. et al. Evaluation between numerical rating scales, visual analog scoring scales, and force plate gait analysis in dogs. Veterinary Surgery, v.36, n.4, p.360-367, 2007. Disponível em: <http://onlinelibrary.wiley.com/doi/10.1111/ j.1532-950X.2007.00276.x/pdf>. Acesso em: 5 jul. 2009. doi: 10.1111/j.1532-950X.2007.00276.x.

MANNE, S.L. et al. Assesment of the acute pediatric pain: do child self-report, parent ratings and nurse ratings measure the same phenomenum? Pain, v.48, p.45-52, 1992. Disponível: $<\mathrm{ht} \mathrm{t}$ : :// w w w. s c i e n c edirect. co m/ 
science?_ob=ArticleURL\&_udi=B6T0K-485H8W7$9 \& \quad$ u s e r $=687358 \&$ c o ver Dat e $=01 \% 2$ F $31 \%$ 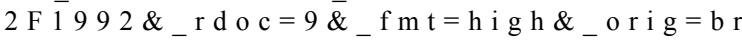 owse\&_origin $=$ browse\&_zone $=$ rslt_list_item \& $s r c h=$ doc info( $\% 23$ toc \% $234865 \% 231992 \% 2399951999$ $8 \% 23405899 \% 23$ F L P \% 23 display\% 23 Volume) \&_cdi $=4865 \&$ _sort $=\mathrm{d} \&$ docanchor $=\&$ \&t $=16 \&$ \& act $=\mathrm{C} 00$ $0037899 \&$ \&ersion $=1 \&$ urlVersion $=0 \&$ \& userid $=68735$ $8 \& \mathrm{md} 5=5 \mathrm{a} 8435672 \mathrm{c} 972 \mathrm{c} 89 \mathrm{~d} 608 \mathrm{c} 6133 \mathrm{aca} 9 \mathrm{fe} 0 \&$ searchtype $=\mathrm{a}>$. Acesso em: 18 jul. 2009. doi: 10.1016/0304-3959(92)90130-4.

MATHEWS, K.A. Pain assessment and general approach to management. Veterinary Clinics of North America: Small Animal Practice, v.30, 729-755, 2000. Disponível em: <http:/ /www.vetsmall.theclinics.com/article/S0195-5616(08)70004-4/ pdf $>$. Acesso em: 30 jul. 2009.

RÉDUA, M.A. et al. The pre-emptive effect of epidural ketamine on wound sensitivity in horses tested by using von Frey filaments.
Veterinary Anesthesia and Analgesia, v.29, n.4, p.200-206, 2002. Disponível em: <http://onlinelibrary.wiley.com/doi/ 10.1046/j.1467-2995.2002.00083.x/pdf>. Acesso em: 5 jul. 2009. doi: 10.1046/j.1467-2995.2002.00083.x.

SELMI, A.L. et al. A comparison of the analgesic efficacy of vedaprofeno, carprofen or ketofen after ovariohysterectomy in bitches. Ciência Rural, v.39, n.3, p.785-790, 2009. Disponível $\mathrm{em}:<\mathrm{http}: / / \mathrm{www}$. scielo.br/scielo.php?script=sci_arttext\&pid=S0103$84782009000300023 \& \operatorname{lng}=$ pt\&nrm=iso\&tlng $=$ en $>$. Acesso em: 24 jun. 2009. doi: 10.1590/S0103-84782008005000104.

VALADÃO, C.A.A. et al. Injeção epidural de morfina ou cetamina em cães: avaliação do efeito analgésico pelo emprego dos filamentos de Von Frey. Arquivo Brasileiro de Medicina Veterinária e Zootecnia, v.54, n.4, p.338-339, 2002. Disponível em: <http:/ /www.scielo.br/scielo.php?script=sci_arttext\&pid=S0102$09352002000400009 \& \operatorname{lng}=\mathrm{en} \& n r m=\mathrm{iso} \& \operatorname{lng}=\mathrm{pt}>$. Acesso em: 24 jun. 2009. doi: 10.1590/S0102-09352002000400009. 